\title{
The Association of Serum Omentin-1 Levels with Severity of Nonalcoholic Fatty Liver Disease in Correlation with Cardiovascular Risk Factors among Egyptian Women
}

\author{
NEARMEEN M. RASHAD, M.D.*; AHMED F. GOMAA, M.D.*; HALA G. ABOMANDOUR, M.D.**; \\ EBTESAM M. IBRAHIM, M.D.*** and MAI M. HASAN, M.D.*** \\ The Departments of Internal Medicine*, Cardiology** and Medical Physiology***, Faculty of Medicine, \\ Zagazig University, Egypt
}

\begin{abstract}
Background: Non-Alcoholic Fatty Liver Disease (NAFLD) is a pathologic condition with insidious course that varies from simple steatosis to steatohepatitis (NASH) with potential progression to cirrhosis. NAFLD is associated with metabolic syndrome, obesity, diabetes mellitus and insulin resistance. Omentin- 1 is an adipokine which possesses anti-inflammatory effects; the role of omentin-1 in NAFLD is scarcely investigated.
\end{abstract}

Aim of Study: Our study aimed to evaluate serum omentin1 levels in women with NAFLD and to assess its relation to the severity of NAFLD and risk factors for cardiovascular morbidities among Egyptian women.

Subjects and Methods: Case control study enrolled 55 women biopsy-proven NAFLD and forty healthy volunteers as a control group. NAFLD patients were divided into three groups; simple steatosis $(n=30)$, NASH $(n=15)$, and cirrhosis $(\mathrm{n}=10)$. Serum omentin-1 levels were measured using an Enzyme-Linked Immunosorbent Assay (ELISA).

Results: Our results revealed that, NAFLD patients had lower values of serum omentin-1 when compared to controls. Among NAFLD patients, patients with cirrhosis had the lowest values of serum omentin-1. We detected significant negative correlations between serum omentin-1 levels and cardiometabolic parameters, obesity indices and liver function among patients with NAFLD.

Conclusion: Women with NAFLD had lower values of serum omentin-1 than control group; moreover, among NAFLD groups, the lowest values were observed in patients with cirrhosis. The diagnostic power of serum levels of omentin1 was highly significant thus; it could represent a promising noninvasive diagnostic biomarker of NAFLD.

Key Words: NAFLD - Omentin-1 - Cardiovascular - NASH - Cirrhosis.

Correspondence to: Dr. Nearmeen M. Rashad, E-Mail: nrashad78@yahoo.com n.rashad@zu.edu.eg

\section{Introduction}

NONALCOHOLIC Fatty Liver Disease (NAFLD) is a major worldwide health problem which represents an epidemic. NAFLD is the most common hepatic disease all over the world that is evidenced by hepatic steatosis, either by imaging or histologypathology, without other causes for secondary fat accumulation in liver like excessive consumption of alcohol, usage of steatogenic medication, or hereditary disorder [1]. NAFLD is a chronic liver disorder which leads to a wide variety of pathological effects such as Non-Alcoholic Steatohepatitis (NASH) and cirrhosis, insulin resistance, obesity, hypertension, dyslipidemia, diabetes and cardiovascular complications [2]

Extensive data support a major role of adipose tissue in pathogenesis of inflammation. Adipose tissue is an endocrine organ that plays a complex but highly active role in homeostasis [3]. It produces various cytokines which are involved in regulating multiple pathologic conditions related to metabolism [4]. The currently reported obesity epidemic is significantly related to the raised prevalence and severity of Non-Alcoholic Fatty Liver Disease (NAFLD); not only obesity is linked to simple steatosis, but also to the progression of the disease [5]

NAFLD has a prevalence of 20-30\% in western countries which is high enough to cause a considerable concern about the progression of this condition from steatosis to steatohepatitis (NASH), cirrhosis, and even hepatocellular carcinoma [6] A growing bulk of evidence supports the "multiple hits" hypothesis which considers multiple insults in subjects who are genetically predisposed to 
NAFLD. It seems more plausible in explaining NAFLD pathogenesis than do other hypotheses. Some of these hits are insulin resistance, adipokines, gut microbiota, nutritional factors, genetic and epigenetic factors. However, the definite molecular mechanisms underlying NAFLD are complex and remain unclear [7].

Omentin-1 is a novel adipokine with high expression in visceral fat. A preponderance of evidence suggests that omentin-1 may regulate insulin resistance by enhancing insulin-stimulated glucose uptake [8] and suppression of vascular inflammation by modulating vascular function and attenuating cyclooxygenase- 2 expression [9].

Taking into consideration the vital role of liver biopsy in diagnosing NASH, and severity of liver disease [10], the need for a novel, simple and non-invasive test which possesses a high accuracy to distinguish NASH from NAFLD and to determine the stage and grade of the disease is increasing [11]. This study was conducted to evaluate serum omentin-1 levels in patients with NAFLD as noninvasive biomarker, and to assess its correlation with severity of NAFLD and cardiovascular risk factors in Egyptian women.

\section{Subjects and Methods}

This study was conducted from January 2017 to January 2018. It is a case control study that included 95 subjects; 55 women biopsy-proven NAFLD and forty healthy volunteers without liver disease, whose Body Mass Index (BMI) and age matching the diseased group, were included as the control group. Patients from Outpatient Clinics of Internal Department, Faculty of Medicine, Zagazig University were recruited. A written, informed consent after explaining details and benefits as well as risks, was taken from all of the participants. Approval of the Ethical Committee of Faculty of Medicine, Zagazig University was obtained.

NAFLD was diagnosed upon ultrasonographic finding of bright liver according to the standard criteria accepted by the American Gastroenterology Association. A confirmatory liver biopsy was done in 30 patients after obtaining a written consent. Subsequently NAFLD patients were divided into three groups; simple steatosis $(n=30)$, NASH $(n=$ $15)$, and cirrhosis $(\mathrm{n}=10)$. Thorough history taking, full clinical examination and anthropometric measures were performed for the studied groups. Dualenergy X-ray absorptiometry (DEXA) scan was done for assessment of Fat Mass Index (FMI) and Fat Free Mas Index (FFMI). Exclusion criteria for all women included any liver disease other than
NAFLD such as hepatitis B or hepatitis C, autoimmune hepatitis, alpha one antitrypsin deficiency or Wilson's disease, alcohol consumption, use of amiodarone, corticosteroids, tamoxifen, methotrexate, oral contraceptives, pregnancy, diabetes, hypertension, thyroid disease, malignancy and decompensated liver disease. All subjects were subjected to abdominal ultrasonography using probe 3.5MHZ of TDI Philips machine. After minimum 8 hours fasting, examination of patients was performed in the supine, right and left lateral positions. Several longitudinal, oblique and transverse scans were performed by a single experienced radiologist in order to avoid the inter-observer variability.

Liver biopsies were obtained using an automated gun device and under complete aseptic precautions. Platelets count and prothrombin concentration were assessed. Pathological examination of liver biopsy was performed by an experienced pathologist who was blinded for patient data. A semiquantitatively evaluation of steatosis, lobular inflammation, ballooning, and liver fibrosis were done according to the NASH CRN scoring system [12] : $\mathrm{F} 0=$ No fibrosis, $\mathrm{F} 1=$ Perisinusoidal or portal/ periportal fibrosis, F2=Perisinusoidal and portal/ periportal fibrosis, $\mathrm{F} 3=$ Bridging fibrosis and $\mathrm{F} 4=$ Cirrhosis. As recently recommended [13]. NASH was defined as the presence of each of the 3 following conditions: Steatosis grade > 1 , lobular inflammation grade $\geq 1$, and ballooning grade $\geq 1$. 'Significant fibrosis' was defined as fibrosis stage F>2.

\section{Laboratory analysis:}

Blood samples were drawn from all after overnight fast. Fasting Plasma Glucose (FPG) was measured calorimetrically with glucose oxidase procedure (Spinreact, Girona, Spain). Total Cholesterol (TC), High Density Lipoprotein (HDL) cholesterol, and Triglycerides (TG) levels were measured using routine enzymatic methods (Spinreact, Girona, Spain). Low Density Lipoprotein (LDL) cholesterol levels were calculated using the Friedewald formula. Liver function tests: Alanine Transaminase (ALT), Aspartate Transaminase (AST), serum bilirubin, serum albumin and Prothrombin Time (PT). Viral markers (HBsAg, HBcAb, HCV Ab), immunoglobulins, ANA, ASMA and anti LKM Ab. Level in plasma by ELISA. Serum ferritin concentrations were measured using (ELISA) kit provided by (Biosource Europe S.A., Nivelles, Belgium). Fasting Insulin (FSI), levels using ELISA kits (DRG International, USA). Insulin Resistance (IR) was calculated with the Homeostatic Model Assessment-IR (HOMA-IR) index, 
which is defined as FSI $(1 \mathrm{U} / \mathrm{mL}) \mathrm{X}$ FPG $(\mathrm{mg} / \mathrm{dl}) /$ 405. Hepatic steatosis index was calculated as: 8 * ALT/AST + BMI (+2, if diabetes; +2 , if female) .

\section{Measurements of serum omentin-1:}

Serum omentin-1 concentrations were determined by Enzyme-Linked Immunosorbant Assay (ELISA) kit (CUSABIO Biotech Co., USA) according to the manufacturer's instructions using ELx808TM Absorbance Microplate Reader, Biotek instruments, USA.

\section{Statistical analysis:}

Data analysis was performed with (SPSS Version 21, Chicago, Illinois). Data were expressed as mean \pm Standard Deviation (SD). Data were tested for normal distribution using the Kolmogorov-Smirnov test. Student's $t$-test and oneway ANOVA with the Turkey's post hoc were performed for variables with normal distribution. Mann-Whitney U and Kruskal-Wallis tests were used for non-normally distribution variables. The relationships of serum omentin-1 levels with clinical and laboratory parameters among patients with NAFLD were tested with the Pearson correlation coefficient. Receiver Operating Characteristic (ROC) analysis was performed to assess the accuracy of serum omentin-1 for diagnosis of NAFLD. A linear regression analysis was done to detect the main predictors of serum omentin-1 levels in NAFLD groups. $p$ is considered to be significant at $<0.05$ with a $95 \%$ Confidence Interval (CI).

\section{Results}

Clinical and biochemical characteristics of the studied groups:

In attempt to compare between control and NAFLD groups we adjusted age and body composition parameters; BMI, waist/hip ratio, FMI\% and FFMI\%. In NAFLD group, we found significant higher levels of systolic blood pressure, TG, LDL, FPG, FSI, and HOMA-IR compared to healthy women. Regarding liver enzymes; AST, ALT and GGT values as we expected they were significantly higher in NAFLD compared to controls. On the contrary, we detected significant lower HDL in NAFLD patients than in those healthy women. Moreover, we observed significant higher levels of hepatic steatosis index in NAFLD cases compared to controls, $p<0.001 *$, (Table 1).

Table (1): Clinical, anthropometric and laboratory characteristics of studied groups.

\begin{tabular}{|c|c|c|c|}
\hline Variables & $\begin{array}{l}\text { Normal group } \\
(\text { mean } \pm \text { SD }) \\
(n=40)\end{array}$ & $\begin{array}{c}\text { NAFLD } \\
(\text { mean } \pm \text { SD) } \\
(n=55)\end{array}$ & $p$ \\
\hline Age (years). & $30.02 \pm 6.37$ & $30.4 \pm 6.91$ & 0.768 \\
\hline Systolic blood pressure (mmHg). & $123.3 \pm 3.07$ & $129.3 \pm 13.1$ & $<0.001 *$ \\
\hline Diastolic blood pressure $(\mathrm{mmHg})$. & $86.1 \pm 4.96$ & $87.9 \pm 7.30$ & 0.156 \\
\hline Body mass index $\left(\mathrm{kg} / \mathrm{m}^{2}\right)$ & $33.6 \pm 4.940$ & $34.3 \pm 3.126$ & 0.126 \\
\hline Waist/hip ratio. & $1.29 \pm 0.194$ & $1.3 \pm 0.168$ & 0.190 \\
\hline FMI $\left(\mathrm{kg} / \mathrm{m}^{2}\right)$ & $6.6 \pm 0.625$ & $6.9 \pm 0.98$ & 0.480 \\
\hline FFMI $\left(\mathrm{kg} / \mathrm{m}^{2}\right)$. & $26.6 \pm 2.50$ & $27.14 \pm 3.95$ & 0.511 \\
\hline Total cholesterol (mg/dL). & $141.3 \pm 26.34$ & $179.6 \pm 51.48$ & 0.479 \\
\hline Triglycerides (mg/dL). & $130.6 \pm 12.51$ & $143.6 \pm 32.01$ & $<0.001 *$ \\
\hline LDL cholesterol (mg/dL). & $122.9 \pm 22.9$ & $156.2 \pm 44.79$ & $<0.001 *$ \\
\hline HDL cholesterol (mg/dL). & $53.1 \pm 6.67$ & $36.9 \pm 6.775$ & $<0.001 *$ \\
\hline Fasting plasma glucose (mg/dL). & $87.9 \pm 3.97$ & $146.1 \pm 51.1$ & $<0.001 *$ \\
\hline Fasting serum insulin $(\mathrm{lU} / \mathrm{mL})$. & $7.6 \pm 2.262$ & $13.2 \pm 9.14$ & $<0.001 *$ \\
\hline HOMA-IR. & $1.67 \pm 0.51$ & $5.3 \pm 4.629$ & $<0.001 *$ \\
\hline Prothrombin time (seconds). & $10.9 \pm 0.23$ & $10.9 \pm 0.245$ & 0.454 \\
\hline AST (IU/L). & $33.3 \pm 3.126$ & $65.5 \pm 20.6$ & $<0.001 *$ \\
\hline ALT (IU/L). & $33.06 \pm 2.5$ & $84.5 \pm 20.67$ & $<0.001 *$ \\
\hline GGT (IU/L). & $32.7 \pm 1.50$ & $79.5 \pm 35.6$ & $<0.001 *$ \\
\hline Serum ferritin $(\mathrm{ng} / \mathrm{ml})$ & $18.02 \pm 3.06$ & $17.02 \pm 4.06$ & 0.902 \\
\hline Hepatic steatosis index. & $34.9 \pm 1.18$ & $46.4 \pm 3.65$ & $<0.001 *$ \\
\hline \multirow{2}{*}{\multicolumn{4}{|c|}{$\begin{array}{ll}\text { FMI } & : \text { Fat Mass Index. } \\
\text { FFMI } & : \text { Fat Free Mass Index. }\end{array}$}} \\
\hline & & & \\
\hline \multicolumn{4}{|c|}{ HOMA-IR : Homeostasis Model Assessments of Insulin Resistance. } \\
\hline \multicolumn{4}{|c|}{$\begin{array}{ll}\text { AST } & \text { : Aspartate Aminotransferase. }\end{array}$} \\
\hline : Alanine Aminotransferase & & & \\
\hline$:$ Gamma-Glutamyltranspep & - 10 a & & \\
\hline
\end{tabular}


Comparison of serum omentin-1 levels $(\mathrm{ng} / \mathrm{ml})$ in the studied groups:

Our results revealed statistically significant lower values of serum omentin-1 in NAFLD (29.3 \pm 7.92) compared to control (50.85 \pm 8.11$)$, Fig. (1A). In addition, the level of serum omentin- 1 were statistically significant lower in cirrhosis group (18.8 \pm 3.084$)$, and NASH group (23.6 \pm 3.49$)$ compared to simple steatosis group $(31.9 \pm 6.89), p$ $<0.001 *$, Fig. (1B).

Clinical and biochemical characteristics of NAFLD groups:

We found statistically significant higher levels of TC, LDL, FPG, FSI, and HOMA-IR in cirrhosis

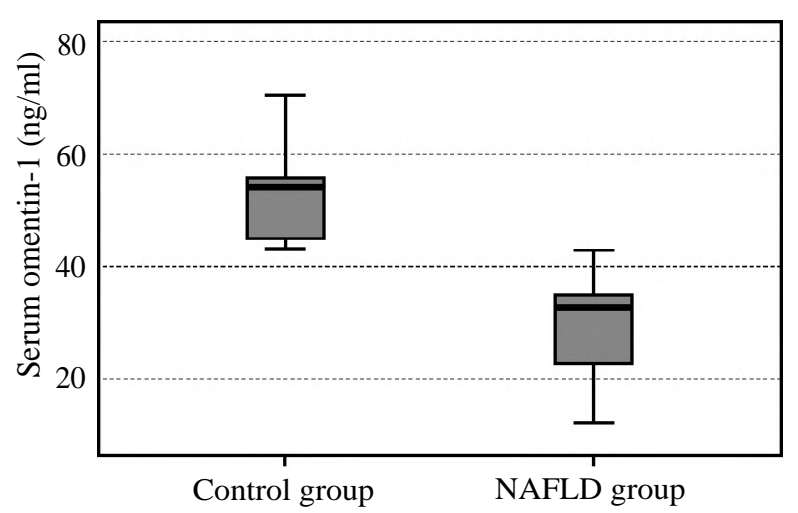

Fig. (1A): Comparison of serum omentin-1 (ng/ml) levels in studied groups. group compared to other groups. However, in NASH group, ALT were significant higher compared to other groups. Moreover, we observed significant lower levels of HDL in cirrhosis group compared to other groups, $p<0.001 *$, (Table 2 ).

Pearson correlation between serum omentin1 levels $(\mathrm{ng} / \mathrm{ml})$ with clinical and laboratory $\mathrm{pa}$ rameters among NAFLD patients:

There were significant negatively correlations between serum omentin-1 levels and body composition parameters; BMI, waist/hip ratio, FMI\% and FFMI\% as well as TG, LDL, FPG, FSI, HOMAIR, and hepatic steatosis index. On the contrary, serum omentin-1 levels were significantly positive correlated with HDL, $p<0.001 *$, (Table 3 ).

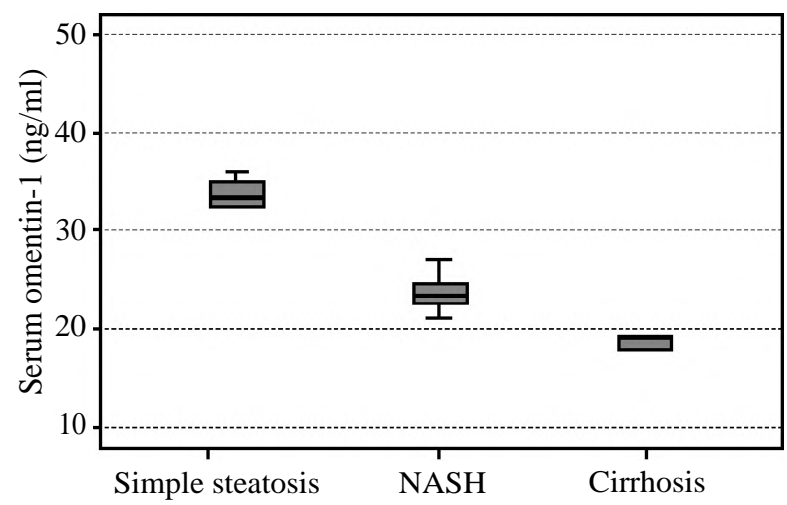

Fig. (1B): Comparison of serum omentin-1 (ng/ml) levels in NAFLD groups.

Table (2): Clinical, anthropometric and laboratory characteristics of NAFLD groups.

\begin{tabular}{lllll}
\hline Variables & Simple steatosis & NASH & Cirrhosis & $p$ \\
\hline Age (years). & $31.5 \pm 7.18$ & $29.2 \pm 4.14$ & $29.1 \pm 9.26$ & 0.464 \\
Systolic blood pressure (mm Hg). & $131.9 \pm 15.57$ & $127.1 \pm 10.4$ & $123.5 \pm 3.37$ & 0.169 \\
Diastolic blood pressure (mm Hg). & $89.1 \pm 8.31$ & $86.6 \pm 6.17$ & $86.1 \pm 5.16$ & 0.374 \\
Body mass index (kg/m ${ }^{2}$ ). & $33.5 \pm 6.33$ & $37.06 \pm 2.98$ & $32.6 \pm 1.71$ & 0.942 \\
Waist/hip ratio. & $1.26 \pm 0.21$ & $1.3 \pm 0189$ & $1.29 \pm 0.144$ & 0.373 \\
FMI (kg/m ${ }^{2}$ ). & $6.8 \pm 1.26$ & $7.61 \pm 0.59$ & $6.52 \pm 034$ & 0.942 \\
FFMI (kg/m ${ }^{2}$ ). & $27.01 \pm 5.06$ & $29.4 \pm 2.38$ & $26.09 \pm 1.36$ & 0.942 \\
Total cholesterol (mg/dL). & $184.4 \pm 61.93$ & $150.4 \pm 22.89$ & $209.1 \pm 16.5$ & $<0.01^{*}$ \\
Triglycerides (mg/dL). & $152.5 \pm 38.2$ & $135.1 \pm 20.7$ & $130 . \pm 12.9$ & 0.072 \\
LDL cholesterol (mg/dL). & $160.4 \pm 53.87$ & $130.9 \pm 19.91$ & $181.9 \pm 14.3$ & $<0.01^{*}$ \\
HDL cholesterol (mg/dL). & $40.4 \pm 4.299$ & $33.6 \pm 6.178$ & $31.6 \pm 8.08$ & $<0.01^{*}$ \\
Fasting plasma glucose (mg/dL). & $125.5 \pm 55.6$ & $178.1 \pm 24.6$ & $160.1 \pm 37.7$ & $<0.01^{*}$ \\
Fasting serum insulin (lU/mL). & $9.7 \pm 4.650$ & $17.3 \pm 9.96$ & $17.9 \pm 13.59$ & $<0.01^{*}$ \\
HOMA-IR. & $3.45 \pm 2.95$ & $7.42 \pm 4.24$ & $7.69 \pm 6.877$ & $<0.01^{*}$ \\
Prothrombin time (seconds). & $10.92 \pm 0.251$ & $10.9 \pm 0.255$ & $10.86 \pm 0.23$ & 0.747 \\
AST (IU/L). & $85.6 \pm 14.13$ & $76.2 \pm 14.42$ & $91.9 \pm 26.31$ & 0.318 \\
ALT (IU/L). & $74.4 \pm 12.7$ & $102.6 \pm 13.3$ & $72.3 \pm 38.2$ & $<0.05^{*}$ \\
GGT (IU/L). & $63.1 \pm 14.62$ & $75.3 \pm 1.76$ & $130.5 \pm 12.6$ & $<0.05^{*}$ \\
Serum ferritin (ng/ml). & $20.4 \pm 2.5$ & $20.3 \pm 2.48$ & $20.25 \pm 2.65$ & 0.973 \\
Hepatic steatosis index. & $45.6 \pm 6.31$ & $51.6 \pm 7.41$ & $42.1 \pm 2.14$ & 0.911 \\
\hline
\end{tabular}

FMI : Fat Mass Index. $\quad$ FFMI : Fat Free Mass Index.

HOMA-IR : Homeostasis Model Assessments of Insulin Resistance. ALT : Alanine Aminotransferase.

AST : Aspartate Aminotransferase. $\quad * \quad: p<0.05$.

GGTP : Gamma-Glutamyl Transpeptidase. 
Table (3): Pearson correlation of serum omentin-1 (ng/ml) with clinical, anthropometric, and biochemical characteristics in NAFLD groups.

\begin{tabular}{llc}
\hline & \multicolumn{2}{c}{ NAFLD $(\mathrm{N}=55)$} \\
\cline { 2 - 3 } Variables & \multicolumn{1}{c}{$r$} & $p$ \\
\hline Body mass index $\left(\mathrm{kg} / \mathrm{m}^{2}\right)$ & -0.58 & $<0.001 *$ \\
Waist/hip ratio & -0.635 & $<0.001 *$ \\
FMI (kg/m ${ }^{2}$ & -0.610 & $<0.001 *$ \\
FFMI (kg/m ) & -0.422 & $<0.001 *$ \\
Total cholesterol (mg/dL) & 0.024 & 0.893 \\
Triglycerides (mg/dL) & -0.692 & $<0.001 *$ \\
LDL cholesterol (mg/dL) & -0.587 & $<0.001 *$ \\
HDL cholesterol (mg/dL) & 0.538 & $<0.001 *$ \\
Fasting plasma glucose (mg/dL) & -0.497 & $<0.001 *$ \\
Fasting serum insulin (lU/mL) & -0.852 & $<0.001 *$ \\
HOMA-IR & -0.714 & $<0.001 *$ \\
Total bilirubin & -0.131 & 0.452 \\
Prothrombin time (seconds) & -0.157 & 0.369 \\
AST (IU/L) & -0.127 & 0.467 \\
ALT (IU/L) & -0.079 & 0.653 \\
GGT (IU/L) & -0.266 & 0.123 \\
Serum ferritin (ng/ml) & -0.079 & 0.653 \\
Hepatic steatosis index & -0.840 & $<0.001 *$ \\
\hline FMI : Fat Mass Index. & & \\
FFMI : Fat Free Mass Index. & & \\
HOMA-IR : Homeostasis Model Assessments of Insulin Resistance. \\
AST : Aspartate Aminotransferase. \\
ALT : Alanine Aminotransferase. \\
GGTP : Gamma-Glutamyltranspeptidase. & \\
$*$ * & & \\
& & \\
\hline
\end{tabular}

A stepwise multiple linear regression analysis in NAFLD group:

In NAFLD group, linear regression analysis revealed that BMI and HOMA-IR were the main predictors of serum omentin-1 levels among other clinical and laboratory biomarkers, $p<0.001 *$, (Table 4).

Accuracy of circulating serum omentin-1 levels $(\mathrm{ng} / \mathrm{ml})$ for diagnosis of NAFLD by ROC analysis:

The power of serum omentin-1 levels (ng/ml) to diagnose NAFLD among studied subjects was evaluated using ROC analysis. The AUC was 0.982 $(95 \% \mathrm{CI}=0.947-1.000)$ with sensitivity $=96.4 \%$, specificity $=97.5 \%$, and the cutoff values (41.3), Fig. (2A).

Accuracy of circulating serum omentin-1 levels $(\mathrm{ng} / \mathrm{ml})$ for differentiate NASH from simple steatosis by ROC analysis:

The power of serum omentin-1 levels (ng/ml) to differentiate NASH from simple steatosis, the AUC 0.873 (95\% CI=0.775-0.970) with sensitivity= $96 \%$, specificity $=56.7 \%$, and the cutoff values (32.9), Fig. (2B).

Accuracy of circulating serum omentin-1 levels $(\mathrm{ng} / \mathrm{ml})$ for differentiate cirrhosis from NASH by ROC analysis:

The power of serum omentin-1 levels $(\mathrm{ng} / \mathrm{ml})$ to diagnose differentiate cirrhosis from NASH, the AUC was 0.863 (95\% CI=0.699-1.000) with sensitivity $=90 \%$, specificity $=73.3 \%$, and the cutoff values (22.5), Fig. (2C).

Table (4): Linear regression analyses in NAFLD women to test the influence of the main independent variables against omentin-1 (ng/ml) (dependent variable) in obese women.

\begin{tabular}{|c|c|c|c|c|c|c|c|}
\hline \multirow{2}{*}{ Model } & \multicolumn{2}{|c|}{ Unstandardized coefficients } & \multirow{2}{*}{$\begin{array}{l}\text { Standardized } \\
\text { coefficients } \\
\text { Beta }\end{array}$} & \multirow{2}{*}{$t$} & \multirow{2}{*}{$\begin{array}{c}p- \\
\text { value }\end{array}$} & \multicolumn{2}{|c|}{$95 \%$ C.I. } \\
\hline & B & Std. Error & & & & Lower Bound & Upper Bound \\
\hline Constant & 6.885 & 1.546 & & 4.452 & 0.000 & 3.777 & 9.993 \\
\hline HOMA-IR & -0.033 & 0.012 & -0.131 & -2.793 & $<0.001 *$ & -0.057 & -0.009 \\
\hline GGT & 0.011 & 0.006 & 0.075 & 1.817 & 0.075 & -0.001 & 0.023 \\
\hline BMI & 1.026 & 0.042 & 0.977 & 24.560 & $<0.001 *$ & 0.942 & 1.110 \\
\hline FSI & 0.038 & 0.090 & 0.067 & 0.424 & 0.673 & -0.143 & 0.220 \\
\hline ALT & -0.039 & 0.179 & -0.034 & -0.215 & 0.830 & -0.398 & 0.321 \\
\hline
\end{tabular}

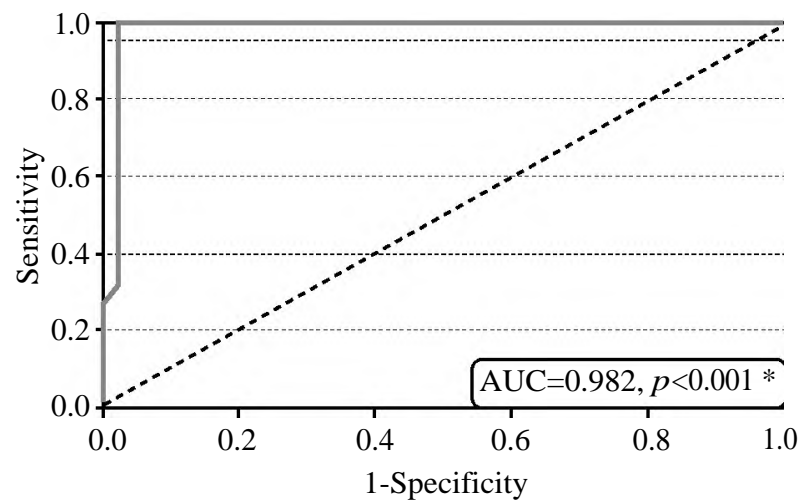

Fig. (2A): Receiver operating characteristic curve of serum omentin-1 for diagnosis of NAFLD.

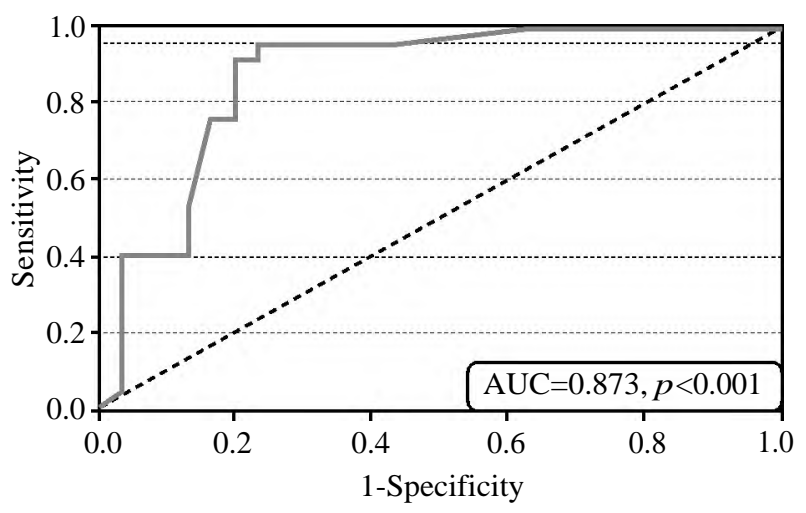

Fig. (2B): Receiver operating characteristic curve of serum omentin-1 for differentiate NASH from simple steatosis. 


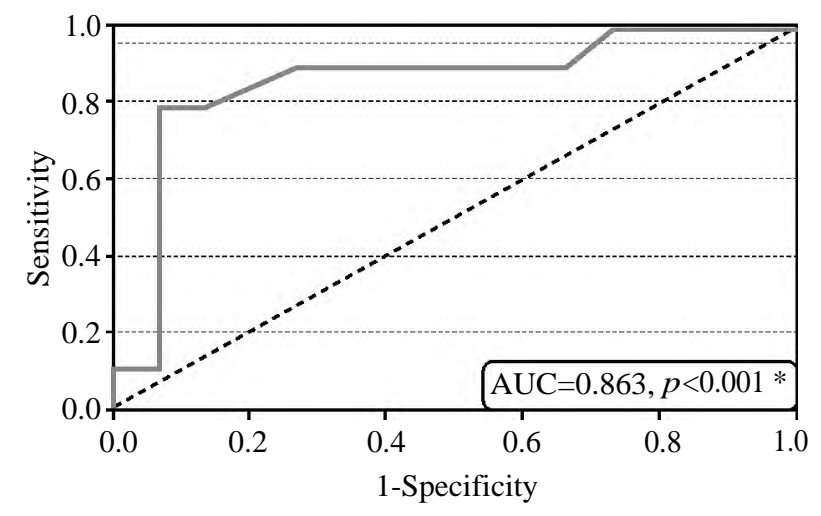

Fig. (2C): Receiver operating characteristic curve of serum omentin-1 for differentiate cirrhosis from NASH.

\section{Discussion}

Obesity is a global health problem with a prevalence of $35 \%$ in females and up to $55 \%$ in South America and the Caribbean, has reached epidemic proportions [14]. Besides being linked with simple steatosis advanced disease, obesity seems to increase liver-specific mortality in NAFLD patients [5].

The global prevalence of NAFLD has been increased from $15 \%$ in 2005 to $25 \%$ in 2010 [15] In Egypt, the prevalence of obesity has reached an alarming rate in the last thirty years affecting $22 \%$ of adult males and $48 \%$ of adult females. It is associated with several comorbidities of particular interest NAFLD [16].

NAFLD comprises a variety of related liver pathologies ranging from simple hepatic steatosis to liver cirrhosis. NAFLD is regarded as an ectopic fat deposition accompanied with metabolic, inflammatory, coagulation and blood pressure disturbances [17].

Hepatic steatosis constitutes the commonest liver disorder in developed countries, with incidence rate of $15 \%-30 \%$ in the general population and $70 \%-90 \%$ in obese individuals or diabetics $[\mathbf{1 8 , 1 9 ]}$

Omentin-1 is a recently identified adipokine that is secreted largely by visceral (omental) fat. As it gains access to the blood; acting as an endocrine factor it accelerates insulin sensitivity, glucose metabolism in muscles, liver and subcutaneous fat [20]. It also possesses a protective role against endothelial dysfunction especially in high-risk patients [21].

Past decades have showed an increased research activity which indicates a key role of inflammation in the pathogenesis of obesity and associated comorbidities. In fact, the etiology of NAFLD is still a mystery however, there are evidences from various studies confirm that NAFLD is a state of low grade inflammation as well as insulin resistance. Hence, we aimed to investigate the serum omentin1 levels in patients with NAFLD, and to assess its correlation with severity of NAFLD and cardiovascular risk factors in Egyptian women.

As expected our results revealed that obesity indices, cardiometabolic parameters, and liver enzymes were significantly high in NAFLD compared to control. An interesting finding of this study was the statistically significant lower values of serum omentin-1 in NAFLD compared to control. Among NAFLD, patients with cirrhosis had the lowest serum omentin-1 levels.

Yilmaz et al., demonstrated elevation of serum omentin-1 levels in patients with fatty liver diseases which was an independent predictor for hepatocyte ballooning in such patients [22].

Our findings disagreed with another Egyptian study conducted on chronic hepatitis $\mathrm{C}$ patients; they found omentin- 1 is elevated in cirrhotic patients [23]. Also, a study conducted by Eisinger et al., on liver cirrhosis patients reported increased omentin-1 levels in absence of complications of portal hypertension [24]. The difference may be due to implication of omentin- 1 in immune and inflammatory response.

Even more importantly, we in this study attempted to pierce out the association of serum omentin- 1 with cardiometabolic parameters, obesity indices and liver function with NAFLD. We detected significant negatively correlations between serum omentin- 1 levels and body composition parameters; BMI, waist/hip ratio, FMI\% and FFMI\% as well as TG, LDL, FPG, FSI, HOMA-IR, and hepatic steatosis index. Regarding HDL, we detected significant positive correlations with serum omentin-1 levels.

These results are in line with the results of de Souza Batista et al., they found decreased levels of omentin-1 in obesity and negative correlation of omentin-1 with BMI, waist circumference, leptin levels, and insulin resistance [25]

In line with our results Yan et al., found low omentin expression in obesity, T2DM and insulin resistance [26] also, Gürsoy et al., study found positive association between serum omentin-1 levels and HDL-C levels in NAFLD patients, suggesting a protective role of omentin-1 in NAFLD [27]. Also, omentin-1 exhibits a protective action 
against visceral obesity and the associated metabolic disturbances [28]

Our findings are in concordance with previous researches, they detected significant negative correlation between omentin- 1 and both fasting insulin levels and HOMA-IR [29].

Similarly, Pan et al., found that circulating omentin-1 levels have been negatively correlated with insulin resistance and obesity [30]

Our study revealed clear evidence that BMI and HOMA-IR were the main predictors of serum omentin-1 levels among other clinical and laboratory biomarkers by linear regression analysis revealed that.

Against our results, Montazerifar et al., found no correlation between serum omentin-1 levels and BMI, whereas a negative correlation between serum omentin-1 and waist/hip ratio, indicating that BMI could be a weaker alternative for body fat distribution than waist circumference was found [29].

Our study found non-significant association between serum omentin-1 and liver enzymes. Similar to our results, Montazerifar et al study reported no correlation between omentin-1 with ALT and AST [29]

Similar to our study, Gürsoy et al., observed positive association between serum levels of omentin-1 and HDL-C levels in NAFLD patients, indicating a protective effect of omentin-1 on NAFLD [27].

Even more importantly, we in this study attempted to pierce out the value of serum omentin1 levels as diagnostic markers of NAFLD by ROC the sensitivity was $96.4 \%$ and the specificity was $97.5 \%$. Considering NASH, the sensitivity was $96 \%$ and the specificity was $56.7 \%$. While the sensitivity $90 \%$ was and the specificity was $73.3 \%$ for differentiating cirrhosis from NASH.

In conclusion women with NAFLD had lower values of serum levels of omentin-1 than control group; moreover, among NAFLD groups, the lowest values were observed in patients with cirrhosis. The diagnostic power of serum levels of omentin1 was highly significant thus; it could be a useful noninvasive diagnostic biomarker of NAFLD.

\section{Conflict of interest: No conflict of interest.}

Acknowledgments: The authors thank doctors of Pathology and Radiodiagnosis Departments,
Faculty of Medicine, Zagazig University for their efforts and help in this research.

\section{References}

1- CHALASANI N., YOUNOSSI Z., LAVINE J.E., DIEHL A.M., BRUNT, E.M., CUSI K., CHARLTON M. and SANYAL A.J.: The diagnosis and management of nonalcoholic fatty liver disease: Practice Guideline by the American Association for the Study of Liver Diseases, American College of Gastroenterology, and the American Gastroenterological Association. Hepatology, 55 (6): pp. 2005-23, 2012.

2- FARZANEGI P., DANA A., EBRAHIMPOOR Z., ASADI M. and AZARBAYJANI M.A.: Mechanisms of beneficial effects of exercise training on non-alcoholic fatty liver disease (NAFLD): Roles of oxidative stress and inflammation. European Journal of Sport Science, pp. 1-10, 2019.

3- BOOTH A., MAGNUSON A., FOUTS J. and FOSTER M.T.: Adipose tissue: An endocrine organ playing a role in metabolic regulation. Hormone molecular biology and clinical investigation, 26 (1): pp. 25-42, 2016.

4- GULCELIK N.E., USMAN A. and GÜRLEK A.: Role of adipocytokines in predicting the development of diabetes and its late complications. Endocrine, 36 (3): pp. $397-$ 403, 2009.

5- POLYZOS S.A., KOUNTOURAS J. and MANTZOROS C.S.: Obesity and nonalcoholic fatty liver disease: From pathophysiology to therapeutics. Metabolism Clinical and Experimental, 92: pp. 82-97, 2019.

6- MAO Y., YU F., WANG J., GUO C. and FAN X.: Autophagy: A new target for nonalcoholic fatty liver disease therapy. Hepatic medicine: Evidence and research, 8, p. 27, 2016.

7- BUZZETTI E., PINZANI M. and TSOCHATZIS E.A.: The multiple-hit pathogenesis of non-alcoholic fatty liver disease (NAFLD). Metabolism, 65 (8): pp. 1038-48, 2016.

8- YANG R.Z., LEE M.J., HU H., PRAY J., WU H.B., HANSEN B.C., SHULDINER A.R., FRIED S.K., McLENITHAN J.C. and GONG D.W.: Identification of omentin as a novel depot-specific adipokine in human adipose tissue: Possible role in modulating insulin action. American Journal of Physiology-Endocrinology and Metabolism, 290 (6): pp. E1253-E1261, 2006.

9- YAMAWAKI H., TSUBAKI N., MUKOHDA M., OKADA M. and HARA Y.: Omentin, a novel adipokine, induces vasodilation in rat isolated blood vessels. Biochemical and biophysical research communications, 393 (4): pp. 668-72, 2010.

10- STRASSBURG C.P. and MANNS M.P.: November. Approaches to liver biopsy techniques-revisited. In Seminars in liver disease (Vol. 26, No. 04, pp. 318-27). Copyright $\odot$ 2006 by Thieme Medical Publishers, Inc., 333 Seventh Avenue, New York, NY 10001, USA, 2006.

11- POYNARD T., MORRA R., INGILIZ P., IMBERTBISMUT F., THABUT D., MESSOUS D., MUNTEANU M., MASSARD J., BENHAMOU Y. and RATZIU V.: Assessment of liver fibrosis: Noninvasive means. Saudi journal of gastroenterology: Official Journal of the Saudi Gastroenterology Association, 14 (4): p. 163, 2008. 
12- KLEINER D.E., BRUNT E.M., VAN NATTA M., BEHLING C., CONTOS M.J., CUMMINGS O.W., FERRELL L.D., LIU Y.C., TORBENSON M.S., UNALP-ARIDA A. and YEH M.: Design and validation of a histological scoring system for nonalcoholic fatty liver disease. Hepatology, 41 (6): pp. 1313-21, 2005.

13- SANYAL A.J., BRUNT E.M., KLEINER D.E., KOWDLEY K.V., CHALASANI N., LAVINE J.E., RATZIU V. and McCULLOUGH A.: Endpoints and clinical trial design for nonalcoholic steatohepatitis. Hepatology, 54 (1): pp. 344-53, 2011.

14- MIRANDA J.J., HERRERA V.M., CHIRINOS J.A., GÓMEZ L.F., PEREL P., PICHARDO R., GONZÁLEZ A., SÁNCHEZ J.R., FERRECCIO C., AGUILERA X. and SILVA E.: Major cardiovascular risk factors in Latin America: A comparison with the United States. The Latin American consortium of studies in obesity (LASO). PloS one, 8 (1): p. e54056, 2013.

15- YOUNOSSI Z.M., KOENIG A.B., ABDELATIF D., FAZEL Y., HENRY L. and WYMER M.: Global epidemiology of nonalcoholic fatty liver disease-meta-analytic assessment of prevalence, incidence, and outcomes. Hepatology, 64 (1): pp. 73-84, 2016.

16- BADRAN M. and LAHER I.: Obesity in Arabic-speaking countries. Journal of Obesity, 2011.

17- SANTOS R.D., VALENTI L. and ROMEO S.: Does nonalcoholic fatty liver disease cause cardiovascular disease? Current knowledge and gaps. Atherosclerosis, 2019.

18- BROWNING J.D., SZCZEPANIAK L.S., DOBBINS R., HORTON J.D., COHEN J.C., GRUNDY S.M. and HOBBS H.H.: Prevalence of hepatic steatosis in an urban population in the United States: Impact of ethnicity. Hepatology, 40 (6): pp. 1387-95, 2004.

19- MARCHESINI G., BUGIANESI E., FORLANI G., CERRELLI F., LENZI M., MANINI R., NATALE S., VANNI E., VILLANOVA N., MELCHIONDA N. and RIZZETTO M.: Nonalcoholic fatty liver, steatohepatitis, and the metabolic syndrome. Hepatology, 37 (4): pp. 917-23, 2003.

20- ELSAID N.H., SADIK N.A., AHMED N.R., FAYEZ S.E. and MOHAMMED N.A.E.G.: Serum Omentin-1 Levels in Type 2 Diabetic Obese Women in relation to Glycemic control, Insulin resistance and Metabolic parameters. Journal of Clinical \& Translational Endocrinology, 13, pp. 14-9, 2018.

21- HAYASHI M., MORIOKA T., HATAMORI M., KAKUTANI Y., YAMAZAKI Y., KURAJOH M., MOTOYAMA K., MORI K., FUKUMOTO S., SHIOI A. and SHOJI T.: Plasma omentin levels are associated with vascular endothelial function in patients with type 2 diabetes at elevated cardiovascular risk. Diabetes research and clinical practice, 148: pp. 160-8, 2019.

22- YILMAZ Y., YONAL O., KURT R., ALAHDAB Y.O., EREN F., OZDOGAN O., CELIKEL C.A., IMERYUZ N., KALAYCI C. and AVSAR E.: Serum levels of omentin, chemerin and adipsin in patients with biopsy-proven nonalcoholic fatty liver disease. Scandinavian Journal of Gastroenterology, 46 (1): pp. 91-7, 2011.

23- NASSIF W.M.H., AMIN A.I., HASSAN Z.A. and ABDELAZIZ D.H.A.: Changes of serum omentin-1 levels and relationship between omentin-1 and insulin resistance in chronic hepatitis C patients. Excli Journal, 12: p. 924, 2013.

24- EISINGER K., KRAUTBAUER S., WIEST R., KARRASCH T., HADER Y., SCHERER M.N., FARKAS S., ASLANIDIS C. and BUECHLER C.: Portal vein omentin is increased in patients with liver cirrhosis but is not associated with complications of portal hypertension. European Journal of Clinical Investigation, 43 (9): pp. 926-32, 2013

25- De SOUZA BATISTA C.M., YANG R.Z., LEE M.J., GLYNN N.M., YU D.Z., PRAY J., NDUBUIZU K., PATIL S., SCHWARTZ A., KLIGMAN M. and FRIED S.K.: Omentin plasma levels and gene expression are decreased in obesity. Diabetes, 56 (6): pp. 1655-61, 2007.

26- YAN P., LIU D., LONG M., REN Y., PANG J. and LI R.: Changes of serum omentin levels and relationship between omentin and adiponectin concentrations in type 2 diabetes mellitus. Experimental and Clinical Endocrinology \& Diabetes, 119 (04): pp. 257-63, 2011.

27- GÜRSOY G., KıRNAP N.G., E SBAH O., ACAR Y., DEMIRBAS B., AKÇAYÖZ S. and ÖZTÜRK A.: The relationship between plasma omentin-1 levels and insulin resistance in newly diagnosed type 2 diabetic women. Clinical reviews and opinions, 2 (4): pp. 49-54, 2010.

28- LI X.P., ZENG S., WANG M., WU X.P. and LIAO E.Y. Relationships between serum omentin-1, body fat mass and bone mineral density in healthy Chinese male adults in Changsha area. Journal of Endocrinological Investigation, 37 (10): pp. 991-1000, 2014.

29- MONTAZERIFAR F., BAKHSHIPOUR A.R., KARAJIBANI M., TORKI Z. and DASHIPOUR A.R.: Serum omentin-1, vaspin, and apelin levels and central obesity in patients with nonalcoholic fatty liver disease. Journal of Research in Medical Sciences: The Official Journal of Isfahan University of Medical Sciences, 22, 2017.

30- PAN H.Y., GUO L. and LI Q.: Changes of serum omentin1 levels in normal subjects and in patients with impaired glucose regulation and with newly diagnosed and untreated type 2 diabetes. Diabetes research and clinical practice, 88 (1): pp. 29-33, 2010. 


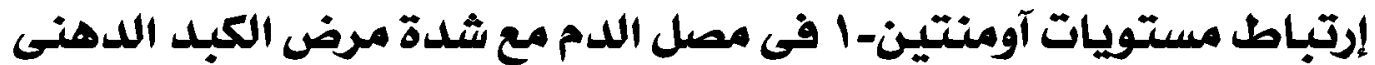

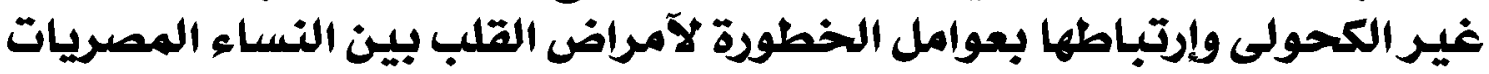

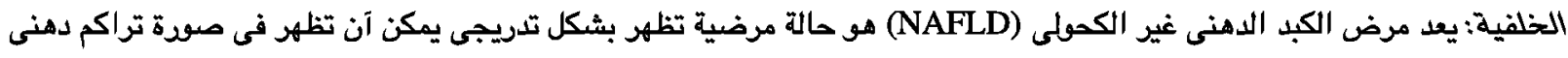

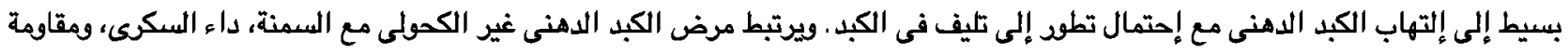

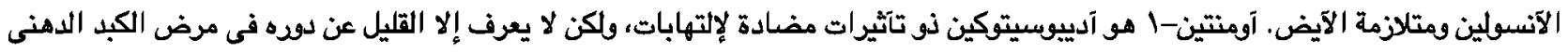

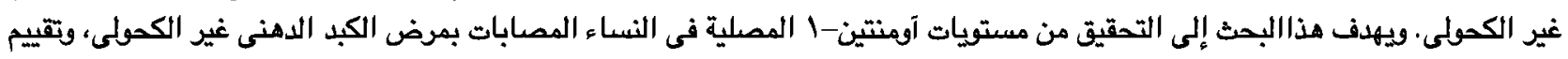

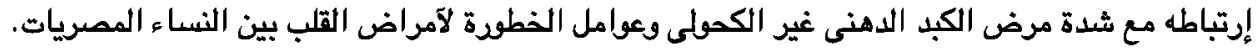

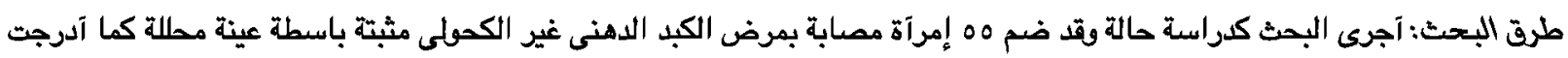

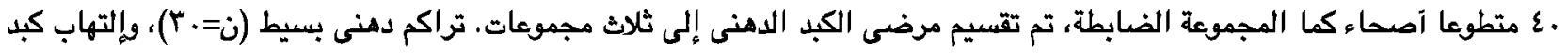

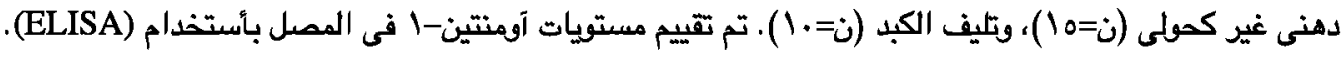

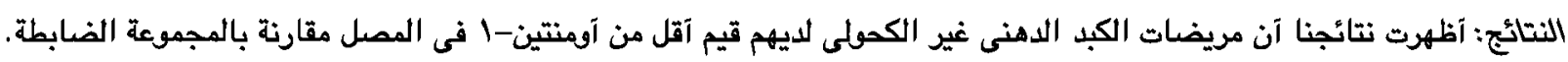

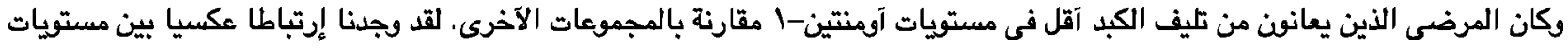

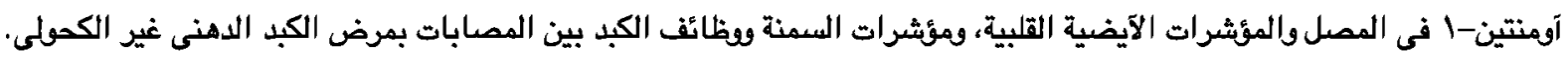

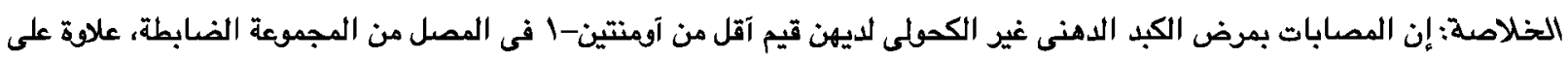

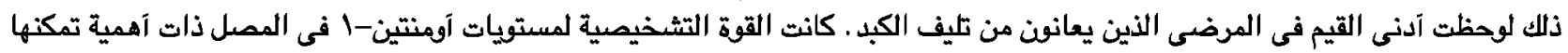

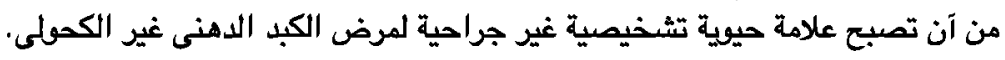

